\section{IN BRIEF}

\section{ANTIMICROBIALS}

\section{Targeting viral liquid-liquid phase separation}

Liquid-liquid phase separation (LLPS) has recently emerged as a novel mechanism of compartmentalization of biomolecules that form membraneless organelles within cells. Studies have shown that several viral proteins and nucleic acids undergo LLPS to form condensates that support viral replication. Now, two recent studies provide evidence that LLPS during viral replication is a target for antiviral therapy. Risso-Ballester et al. discovered that a steroidal alkaloid cyclopamine and its chemical analogue (A3E) inhibit respiratory syncytial virus (RSV) replication by disorganizing and hardening viral inclusion body (IB) condensates, where viral RNA synthesis occurs. In untreated infected cells, viral IBs had a dynamic nature and the RSV phosphoprotein had a liquid-like behaviour, consistent with the formation of viral IBs through LLPS. In compound-treated cells, IB disorganization occurred within minutes, suggesting that these molecules directly inhibit the liquid properties of RSV IBs. The authors also demonstrated that A3E suppresses viral replication in RSV-infected mice. In a separate study, Wang et al. found that targeting LLPS of the SARS-CoV-2 nucleocapsid protein (NP) with a peptide promotes innate antiviral immunity in vitro and in vivo. Previous work showed that NP phase separates into condensates that contain viral RNA and the viral RNA polymerase. In this study, Wang et al. found that the NP dimerization domain (DD) is required for NP to undergo LLPS with RNA, which prevents Lys63-linked poly-ubiquitination and aggregation of mitochondrial antiviral-signalling protein (MAVS), thus suppressing innate immunity in infection. Using a screening approach, the authors identified a peptide that targets the DD. Remarkably, treatment with the peptide led to the disruption of NP LLPS and increased innate antiviral responses in vitro and in mice. Together, these two studies highlight the potential of targeting LLPS to treat viral infections by disrupting viral replication or boosting host immunity.

ORIGINAL ARTICLES Risso-Ballester, J. et al. A condensate-hardening drug blocks RSV replication in vivo. Nature https://doi.org/10.1038/s41586-021-03703-z (2021) | Wang, S. et al. Targeting liquid-liquid phase separation of SARS-CoV-2 nucleocapsid protein promotes innate antiviral immunity by elevating MAVS activity. Nat. Cell Biol. https://doi.org/10.1038/s41556-021-00710-0 (2021)

\section{BACTERIAL PHYSIOLOGY}

\section{Sensing when to colonize}

Members of the gut microbiome originate from external sources such as the environment, food and other individuals, but how individual microbial constituents regulate their transmission into new hosts is not well-explored. In a recent study, Robinson et al. uncover that sensing of host amino acids in Aeromonas veronii regulates bacterial motility, enhancing gut colonization of zebrafish. Using a tractable experimental evolution model in gnotobiotic zebrafish, the authors identified traits in A. veronii that promoted host colonization, including mutations in a gene that encodes an amino-acid-sensing diguanylate cyclase (spdE). Interestingly, loss of spdE in $A$. veronii led to rapid immigration into zebrafish and fish-to-fish transmission. The authors found that SpdE predominantly recognizes the amino acid proline as well as valine and isoleucine, leading to a reduction in the synthesis of cyclic-di-GMP, an intracellular second messenger that regulates bacterial motility. Notably, binding of amino acids to SpdE increased bacterial motility through chemotaxis and chemokinesis, and bolstered host colonization. The authors also observed that bacterial colonization and SpdE-dependent motility was regulated by the resident microbiota, as bacterial collagenolytic activity can liberate SpdE ligands from the host, thus increasing bacterial motility and enhancing host transmission. ORIGINAL ARTICLE Robinson, C. D. et al. Host-emitted amino acid cues regulate bacterial chemokinesis to enhance colonization. Cell Host Microbe https://doi.org/ 10.1016/j.chom.2021.06.003 (2021)

\title{
Arming the host
}

Microorganisms produce numerous specialized metabolites, and the pathways required for the synthesis of these metabolites are encoded by biosynthetic gene clusters (BGCs). Microbial metabolites have important roles in ecological interactions, and various potential industrial and medical applications. For example, bacteriocins are antimicrobial peptides that are produced by many bacteria to inhibit competing strains. Indeed, often these specialized metabolites exert antimicrobial activity, which indicates that they have a crucial role in competitive interactions. However, although much progress has been made in identifying the various types of BGCs and the producing microorganisms, the natural functions of specialized metabolites or their prevalence in microbial communities is less well understood. Interestingly, BGCs have been found in phage genomes, in particular in temperate phages or prophages, which suggests an ecological role of BGC exchange between bacterial strains. In this study, Dragoš, Strube and colleagues report that bacteriocins are encoded in temperate phages, and that they can be transferred between bacterial hosts, which provides a competitive advantage.

First, the authors assessed the prevalence of BGCs that encode specialized metabolites in phage

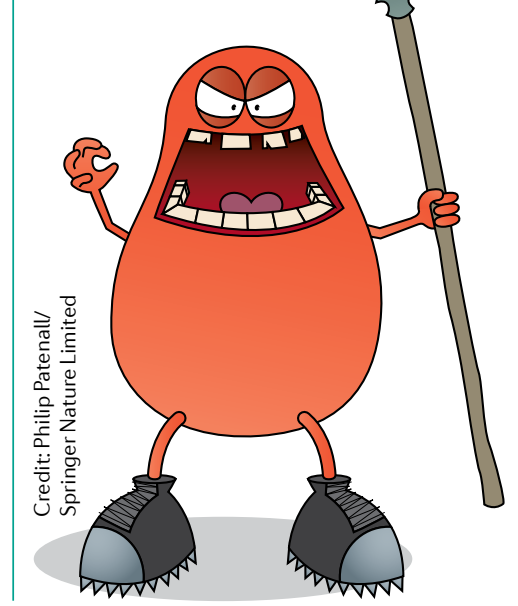

genomes. The analysis revealed that phage-encoded BCGs are very rare, and most of those clusters were found to reside within hostassociated temperate phages that infect particular commensal or pathogenic bacterial species. Interestingly, the authors found that almost all phage-encoded BGCs carried core genes for the biosynthesis of bacteriocins, and they hypothesized that this might confer a fitness advantage to their hosts during inter-species competition. To test this notion, the authors focused on the SP $\beta$ prophage, which is present in some strains of Bacillus subtilis and produces the bacteriocin sublancin. They used isolated SP $\beta$ virions to infect non-lysogen receiver strains that lacked the SP $\beta$ phage, and showed that the newly infected strain was able to outcompete the ancestor strain. Transfer of the phage also occurred during co-culture, which was evidenced by the integration of SP $\beta$ and antimicrobial activity in the receiver strain. The results are in agreement with the hypothesis that a phage that carries a BGC can confer a fitness benefit to its host bacterium through lysogeny.

Finally, the authors assessed the role of phages in the horizontal gene transfer of BGCs between closely and more distantly related bacteria, and they found that transfer within a genus occurred much more frequently than transfer between different host genera.

In sum, the findings demonstrate a natural role of phage-encoded BGCs in inter-bacterial interactions, and that the prophage provides a fitness advantage to its bacterial host, and further suggest that a temperate strategy is advantageous. 